\title{
Probiotics supplementation and insulin resistance: a systematic review
}

\author{
Bárbara Izabel Moraes Salles, Débora Cioffi and Sandra Roberta G. Ferreira* (D)
}

\begin{abstract}
Background: Research on intestinal microbiota has grown considerably, as well as the interest on probiotics' supplementation effects on metabolism. Considering high prevalence rates of metabolic diseases linked by insulin resistance, we performed a systematic review of existing literature which addressed the role of probiotics in modulating insulin sensitivity in animals and humans.

Methods: This systematic review was based on PRISMA guidelines. Searches for original articles published in English from 1990 to January 2020 were made in the electronic database of PubMed from the National Library of Medicine, using Medical Subject Headings to identify longitudinal studies conducted in animals and humans which reported effects of probiotics in a variety of insulin resistance parameters.
\end{abstract}

Results: Overall, results from 27 probiotic interventions (Lactobacillus, Bifidobacterium, Clostridium and Akkermansia) indicated significant beneficial changes in insulin resistance measures in animal studies. Additionally, they improved lipid profile, inflammatory and oxidative markers, short-chain fatty acids production and microbiota composition. In seven clinical trials, samples and designs were heterogeneous. Five showed benefits in insulin resistance parameters and in two others no effect was detected.

Conclusion: Available data regarding the effects of certain probiotics do not guarantee sustained amelioration of insulin resistance in humans. Consistent beneficial results for intestinal barrier function, immune system and metabolism were reported in animals may encourage long-term randomized clinical trials in people with obesity and cardiometabolic risk. Whether supplementation with probiotics in combination with medications and/or prebiotics, associated with a healthy lifestyle, will prove useful to attenuate insulin resistance requires further investigation.

Keywords: Probiotics, Gut microbiota, Endotoxemia, Insulin resistance, Type 2 diabetes mellitus, Prediabetes, Inflammation, Short-chain fatty acids

\section{Introduction}

Growing rates of obesity represent a global lifestyle-related health problem [1]. Its presence favors comorbidities and increases mortality mainly due to cardiometabolic complications [1]. In particular, visceral adipose tissue depot produces inflammatory cytokines inducing insulin resistance, involved in the

*Correspondence: sandrafv@usp.br

Departamento de Epidemiologia, Faculdade de Saúde Pública, School of Public Health, University of Sao Paulo, Av. Dr. Arnaldo, 715, São Paulo, SP CEP 01246-904, Brazil pathophysiology of type 2 diabetes mellitus (DM), hypertension and dyslipidemia; this condition increases the risk of cardiovascular disease, the leading cause of death in most populations [2]. DM is also among the top ten causes of death; it is estimated that 463 million adults have DM and 374 million prediabetes worldwide and the number of diabetic people is predicted to increase to 700 million in 2045 [3]. This scenario has justified the search for underlying mechanisms of these lifestyle-related diseases.

Studies in animals and humans have emphasized the role of gut microbiota as a mediator of cardiometabolic 
disorders [4-6]. Trillions of microorganisms inhabit the gastrointestinal tract, exceeding the number of human cells by ten times [6]. They play a crucial role in immunity, nutrients' absorption, bioactive molecules actions and in metabolism homeostasis [4, 7]. Diversity and stability of the microbiota are affected by genetic and environmental factors. An imbalance of bacterial communities is called dysbiosis that has been described in obesity and other states of insulin resistance [8].

Animal models provided initial evidence of differences in microbiota composition according to body adiposity. Predominance of the phylum Firmicutes was reported in obese mice [9]. Transplantation of fecal microbiota from lean donors to obese animals revealed the ability of bacterial content in changing body adiposity and related disorders in recipients [9-11]. In obese humans, a higher proportion of Firmicutes relative to Bacteroidetes was also described, although heterogeneous findings were further reported $[4,9]$. Promising results of fecal transplantation have also emerged from clinical trials including obese individuals with metabolic disturbances [9].

Beyond the taxonomic classification, some authors have proposed describing clusters of bacterial genera named enterotypes [12]. Western diet, rich in protein and saturated fatty acids, was associated with the Bacteroides enterotype, while diet rich in complex carbohydrates and vegetables with the Prevotella enterotype [13].

Diets enriched with saturated fatty acids have been associated with increased abundance of gram-negative bacteria containing lipopolysaccharides (LPS) on their surface. This condition augments intestinal permeability and LPS translocation, leading to metabolic endotoxemia, inflammation and insulin signaling deterioration $[5,6,9,14]$. On the other hand, fiber-rich diets have been associated with beneficial effects on energy balance [15]. Short-chain fatty acids (SCFA) derived from fiber fermentation acting on enteroendocrine cells resulting in local and remote benefits [16]. Gut-derived butyrate, acetate and propionate play a role in central regulation of satiety and metabolism and have been considered a relevant pathophysiological link between gut microbiota and disturbances of glucose metabolism [15]. In individuals with and without type $2 \mathrm{DM}$, comparisons of microbiota composition have shown lower abundance of a butyrateproducing bacterium and Akkermansia muciniphila, in diabetics $[9,14]$, while in mice dietary supplementation with butyrate ameliorated inflammation and insulin resistance [16].

Evidence on the potential of gut microbiota to improve inflammation and metabolic disturbances in diabetic mice [16] raised the hypothesis that probiotics could represent a therapeutic tool to attenuate microbiota-related mechanisms that generate insulin resistance in human diseases [5, 6]. Probiotics are preparations of microbial cells or their components that confer health benefits to the host when administered in adequate amounts. They are generally safe for human consumption and act through the microbiota modulation. Among potential benefits, those on improving glucose metabolism have been widely studied [17]. Considering the deleterious effects of Western diet in intestinal permeability to endotoxins [9], probiotics have been proposed to improve in microbiota composition. Evidence has indicated that intestinal barrier can be restored by increasing tightjunction proteins expression which reduces endotoxemia and ameliorates insulin sensitivity.

Based on results obtained from animal models and preliminarily in humans [18], probiotic interventions have been tested as a potential strategy for the prevention or treatment of type 2 DM. Several bacterial strains have been proposed to enhance abundance of bacteria which mitigate inflammation and insulin resistance, thus protecting against cardiometabolic diseases. Most studied interventions were based on Lactobacillus, Bifidobacterium and Akkermansia muciniphila [6, 9]. Akkermansia genus has the ability to breakdown mucins from the mucus layer providing oligosaccharides sources to be metabolized by resident microbiota.

Animal studies have demonstrated that obesity and DM induced by high-fat diet (HFD) is at least partially mediated by the gut microbiota. Supplementation of Bifidobacterium breve promoted less weight and visceral adipose tissue gains and improved lipid and glucose metabolism [19], while Bifidobacterium animalis was able to reverse the HFD-induced DM [20]. Animal studies with probiotics addressed to obesity-related outcomes have employed mainly Lactobacillus and Bifidobacterium species based on heterogeneous research protocols [21]. Experiments with Lactobacillus and Bifidobacterium mixture administered in humans resulted in improvement of insulin sensitivity $[17,22]$. As a proof of concept, a small study in humans revealed encouraging results by the administration of live or pasteurized A. muciniphila [23].

The predominance of studies regarding probiotics effects on metabolism were conducted in animals. Supplementations were made with diverse bacteria in different amounts and different durations limiting comparison of their potential benefits. Experimental protocols also differed regarding the type of culture, formulations and storage conditions that could generate responses [46]. Protective cardiometabolic effects of probiotics in humans with or at-risk for cardiometabolic diseases were less investigated $[5,9,24]$. As far as we know, no systematic review in this issue included experiments conducted in animals as well as in humans. 
Considering the burden of cardiometabolic diseases, the potentialities of probiotics supplementation as adjuvant tools for their control and the heterogeneity of the reported studies, a systematic review could indicate important gaps in this knowledge and orient further research. Although changes in gut microbiota composition by probiotics seem an attempt to minimize inflammation and insulin resistance, it is unclear whether they could attenuate such underlying mechanisms of lifestyle-related diseases highly prevalent in humans. Since modern dietary habits are determinants of metabolic disturbances, interventions on diet using probiotics' supplementation represent an opportunity for prevention of insulin resistance-linked diseases. We performed a systematic review of existing literature in English which addressed the role of probiotics in modulating insulin sensitivity in animals and humans.

\section{Methods}

This review was performed based on the PRISMA guidelines (Preferred Reporting Items for Systematic Reviews and Meta-Analysis). Detailed information is provided in Additional file 1.

\section{Search strategy}

Searches for original research articles offered online, published in English from 1990 to January 2020, were conducted in the electronic database of PubMed from the National Library of Medicine. The following Medical Subject Headings (MeSH) were used in several combinations: "probiotics", "lipopolysaccharides", "gastrointestinal microbiome", "intestinal mucosa", "endotoxemia", "fatty acids, volatile", "lactobacillus", "propionibacterium", "bifidobacterium", "saccharomyces", "insulin resistance", "insulin sensitivity", " type 2 diabetes", "type II diabetes", "prediabetes" and "glucose intolerance". The terms "akkermansia" and "enterotype" are not MeSH descriptors although they were also searched considering their importance for this review's purpose. Reference lists of included studies were additional sources.

\section{Eligibility criteria}

Inclusion criteria were all longitudinal controlled studies with probiotics supplementing diet of animal models or humans and an available objective measurement of insulin sensitivity or resistance. Exclusion criteria were participants with type $1 \mathrm{DM}$, pregnant women, lack of information regarding the intervention with probiotics and review studies.

\section{Data handling}

Two independent reviewers (BIMS, DC) assessed the titles and abstracts of all retrieved references to identify studies that potentially met eligibility criteria and eligible articles were retrieved in full text. If duplication of the same study was found, its data were included just once. Disagreements were solved through discussion with another reviewer (SRGF). All relevant study data were extracted into a Microsoft Excel database. Variables extracted included the following: year of publication, country of research, sample characteristics (type and size), experimental conditions (study design and protocol), probiotic intervention (strain type, doses, duration), primary parameter of insulin sensitivity/resistance (baseline and post-intervention) and secondary parameters. Double entry was used to assure the accuracy of data included in the database. These data were summarized in tables, for animal models and humans separately.

The flowchart of articles selected are shown in Fig. 1. Sum of MeSH indexed search and free search on PubMed database returned 86 articles; 30 appeared in duplicate and were excluded, resulting in 56 articles for this review. Initial screening of abstracts was made in 56 articles conducted in animals and humans. Among those, seven review articles were excluded. A total of 49 studies had full text reading by two reviewers (BIMS; DC) and from these, 16 were excluded due to lack of an objective measure of insulin resistance; not being a probiotic; and not analyzing the effect of the probiotic, resulting in 33 studies included in our systematic review. Further, one recent article [23] that was not detected using the defined MeSH but was automatically delivered (Google Scholar Alerts) to one author (SRGF) was also included.

A total of 34 articles were analyzed, distributed in 27 studies involving animal models and seven clinical trials. Probiotics used in the interventions (mainly Lactobacillus and Bifidobacterium) have already recognized benefits on health. Most studies (82\%) were carried out in the last 5 years in different continents. Studies' origin was concentrated in Asia (China, Japan, Korea and India), Europe (France, Spain, Sweden and Austria) and one country in Latin America (Brazil).

The totality of animal studies included in this review was conducted in rodents. In the majority of these experiments HFD was used to induce obesity and insulin resistance and different probiotics were chosen to evaluate their performance on several metabolic parameters. One study [25] used high-fructose diet to induce obesity and glucose intolerance and another maintained a normal chow diet [26]. In clinical trials, no special dietary recommendation was made. All trials were randomized and controlled, being six double-blinded and one single-blinded, four were multicentric and three were single-center. Sample characteristics were diverse, having included healthy participants as well as individuals with type $2 \mathrm{DM}$ or metabolic syndrome (MS). 


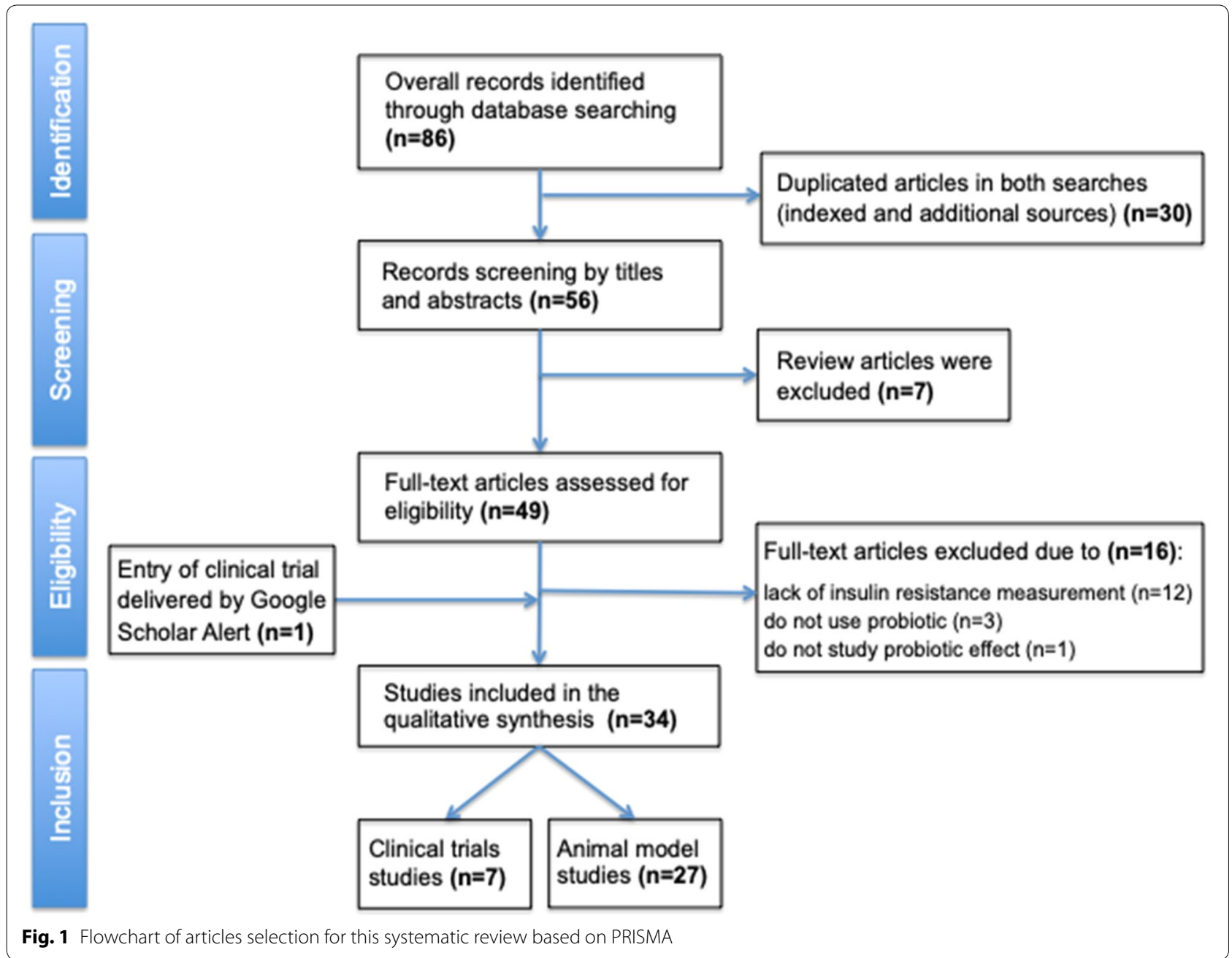

\section{Analysis and interpretation}

Main details of the studies selected including probiotic strains, doses and other experimental characteristics, effects of probiotic interventions on parameters of glucose metabolism and other relevant outcomes are shown in Tables 1 and 2.

Reviewer risk of bias was minimal due to strict collection of eligible articles from literature and because only statistically significant findings were considered to interpret the study result by three investigators. The only exception and possible source of bias could be related to inclusion of a recent small clinical trial with A. muciniphila by Depommier et al. [23]. Despite its preliminary nature, authors agreed about the relevance of this inclusion based on promising results for clinical practice. There was always a consensus among authors in relation to interpretation of the effects of all interventions in parameters of insulin sensitivity or resistance provided in the studies analyzed.

\section{Results}

Animal studies

Main results related to glucose metabolism of 27 animal studies are shown in Table 1 and additional findings are mentioned in the text. The majority of them was performed using strains of Lactobacillus $(\mathrm{n}=20)$ and Bifidobacterium $(\mathrm{n}=4)$, as single-strain probiotics or blends of multiple strains (Lactobacillus + Bifidobacterium). Two studies used Akkermansia and only one used Clostridium. Few studies reported findings regarding microbiota, presenting proportions of bacteria belonging to the main phyla; sometimes, the ratio was mentioned as Firmicutes-to-Bacteroidetes and others Bacteroidetes-toFirmicutes. In four articles, effects of probiotics were compared to oral hypoglycemic drugs (metformin, pioglitazone and vildagliptin).

Except for one 4-week study [27], all probiotic interventions had significant results in at least one measure of insulin resistance. In all studies using single-strain 


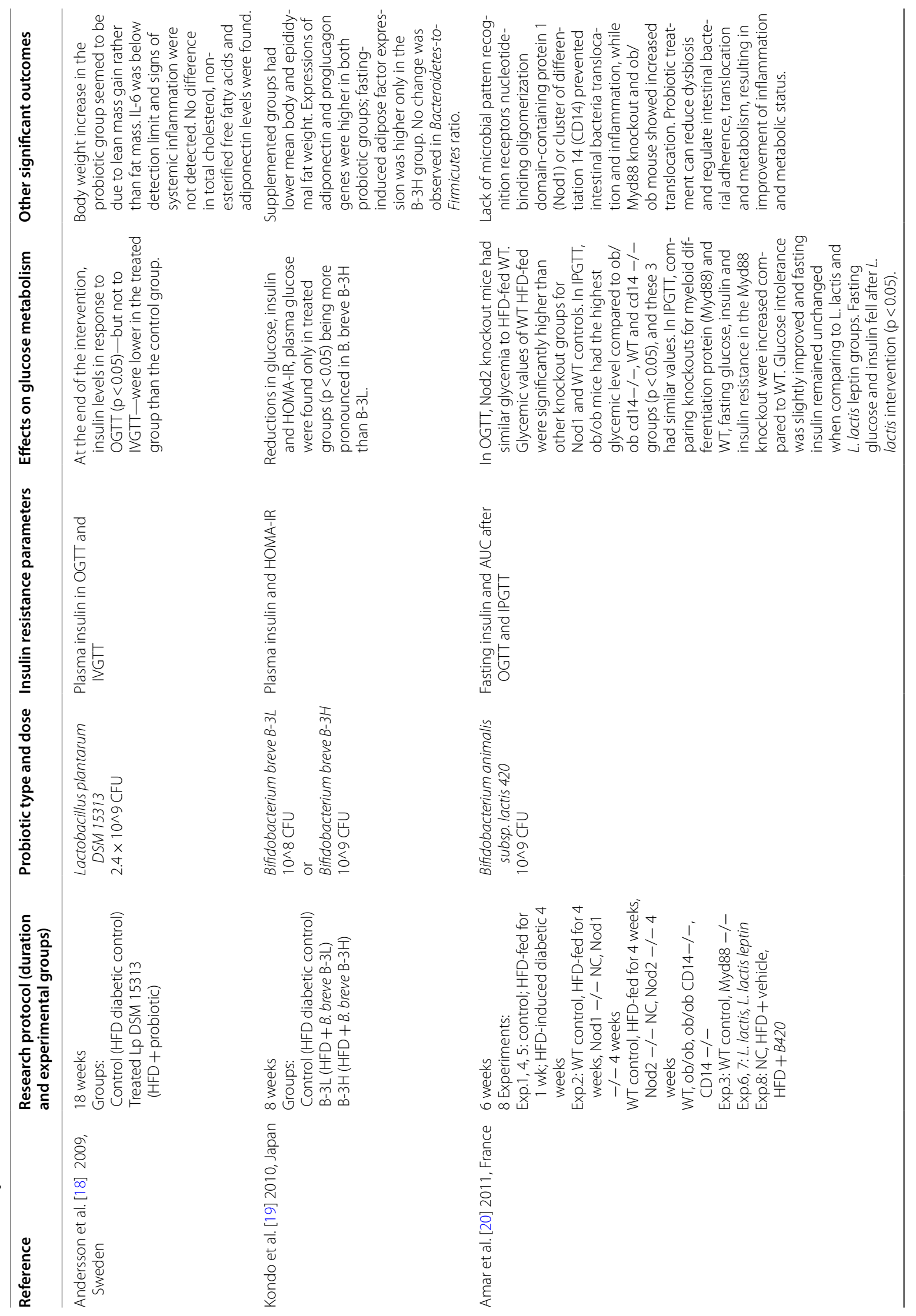




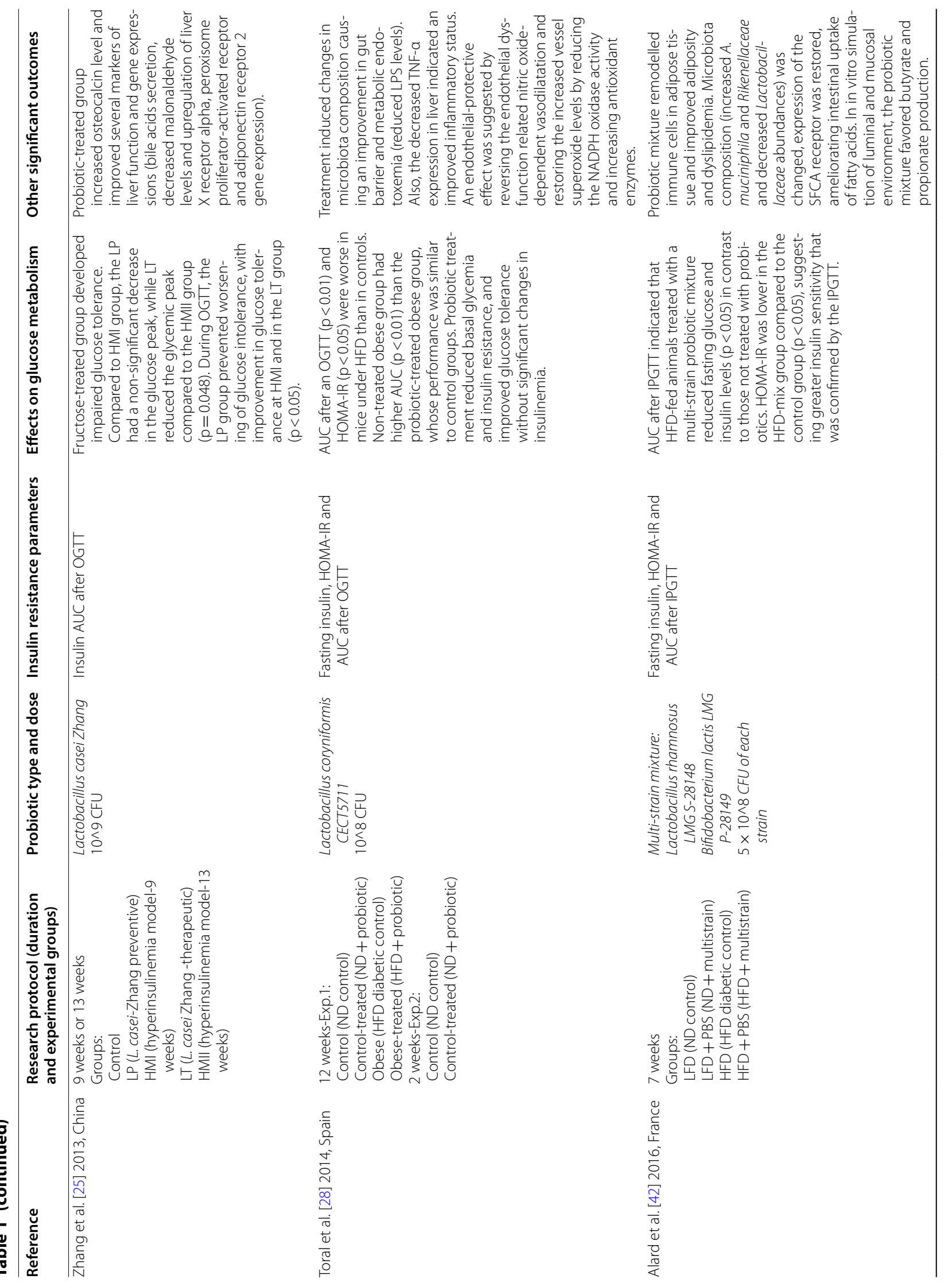




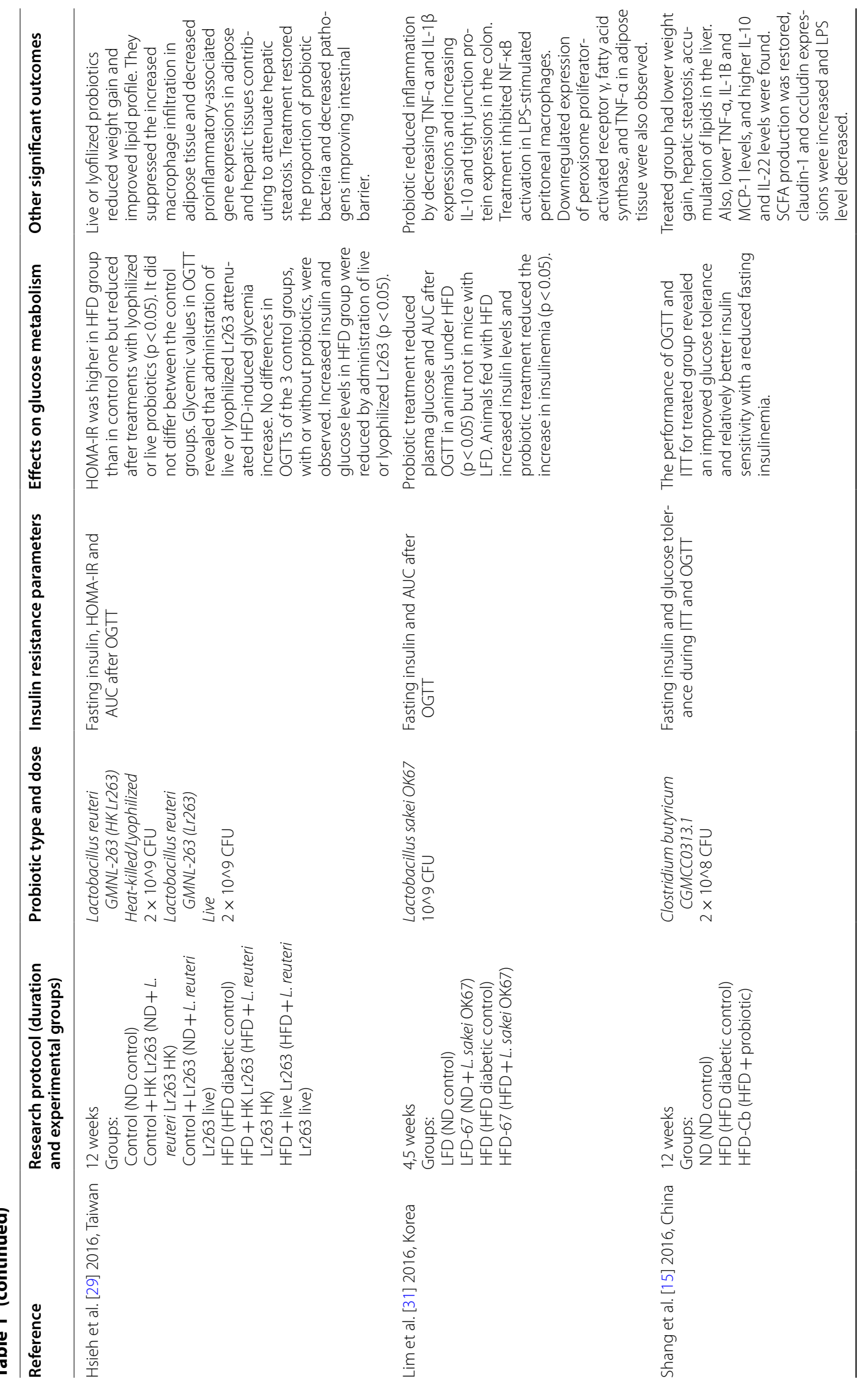




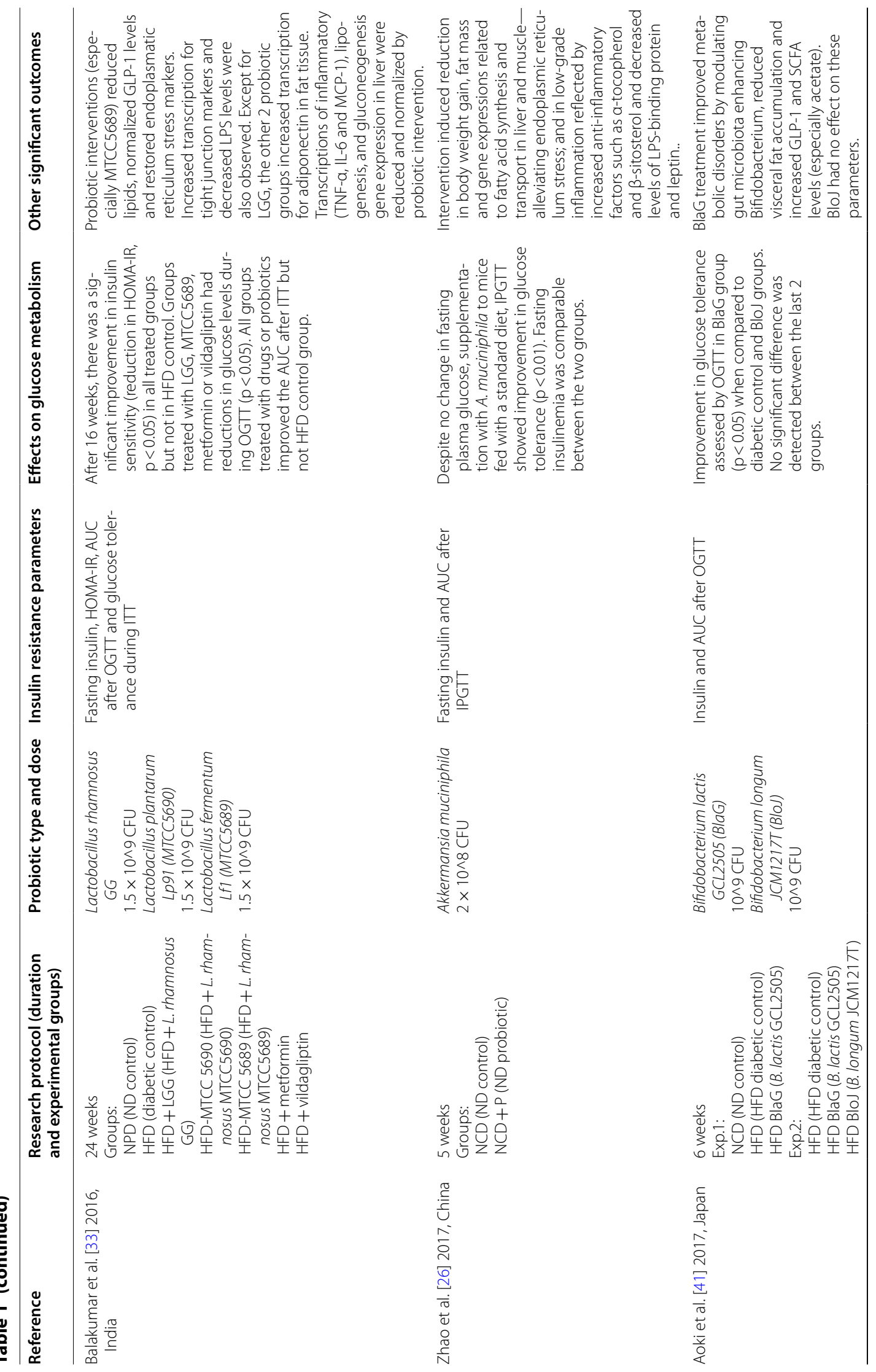




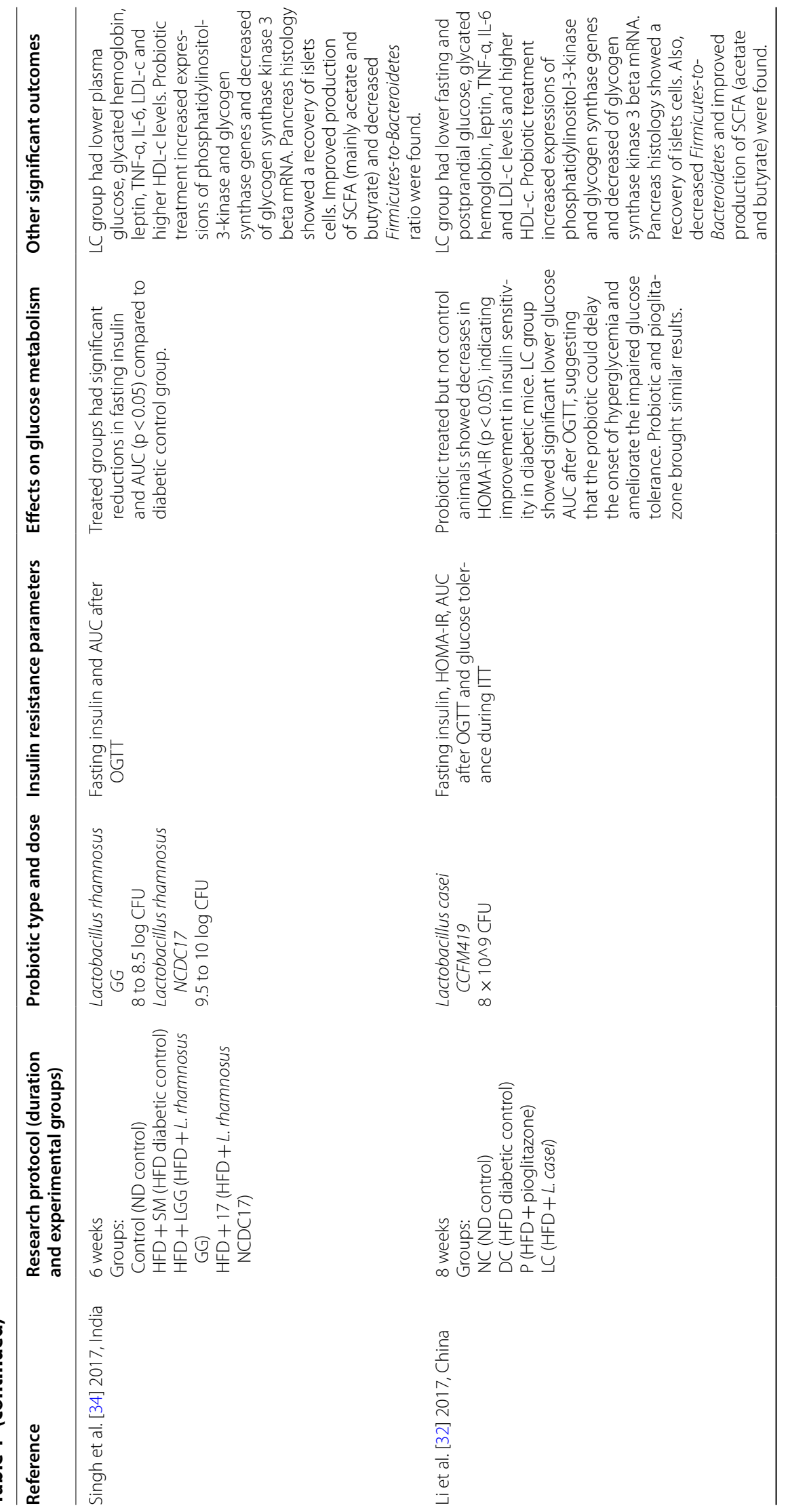



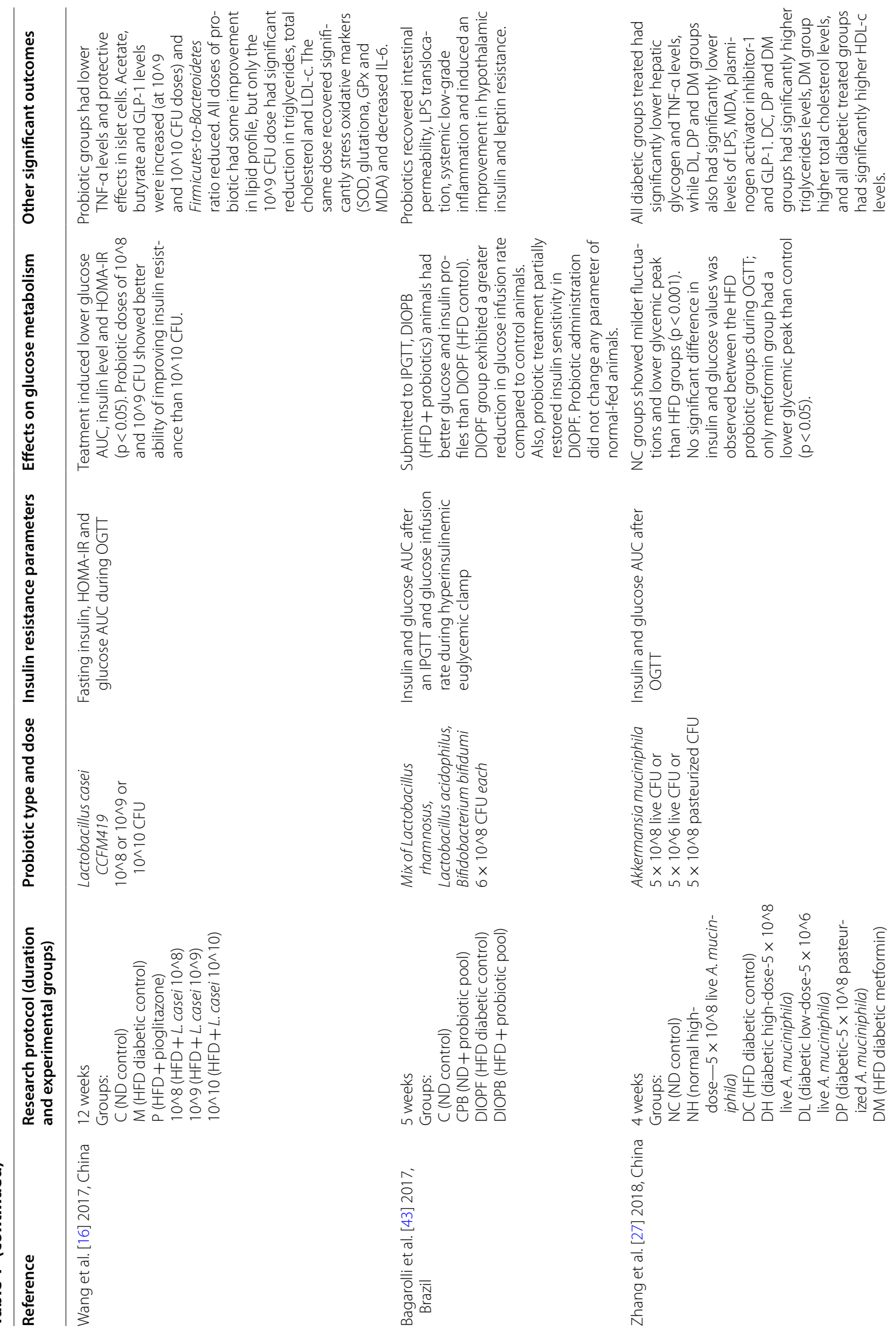


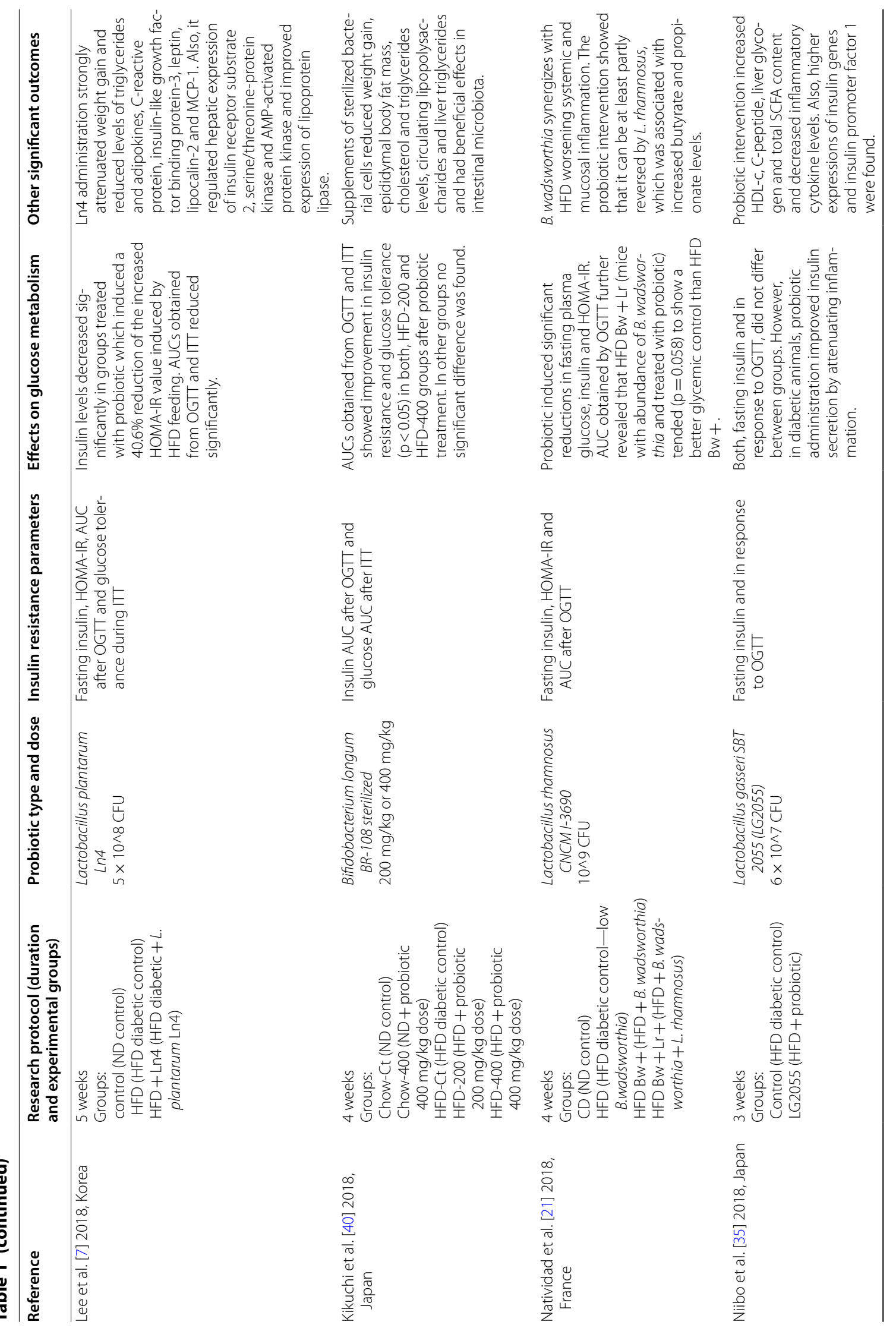




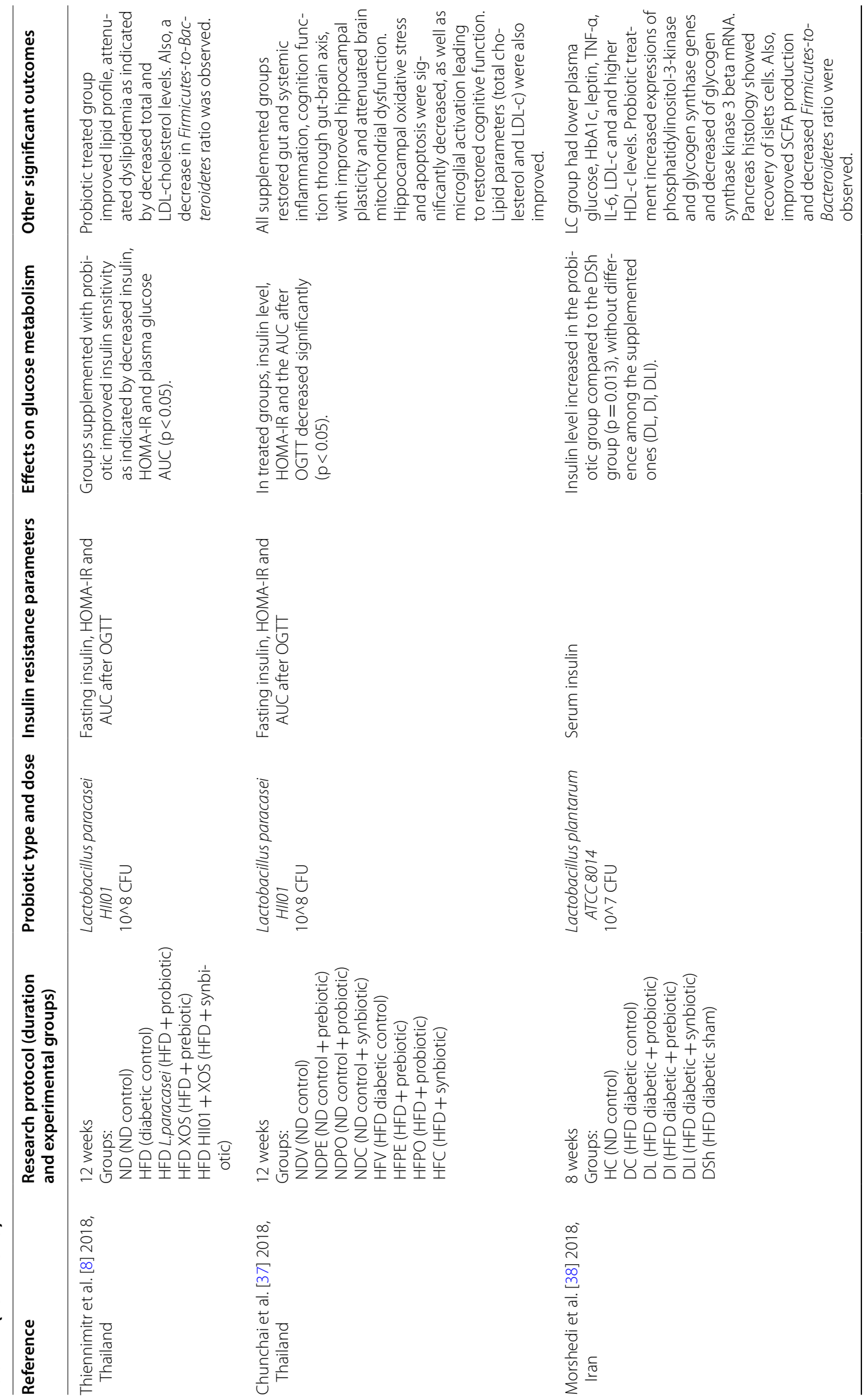




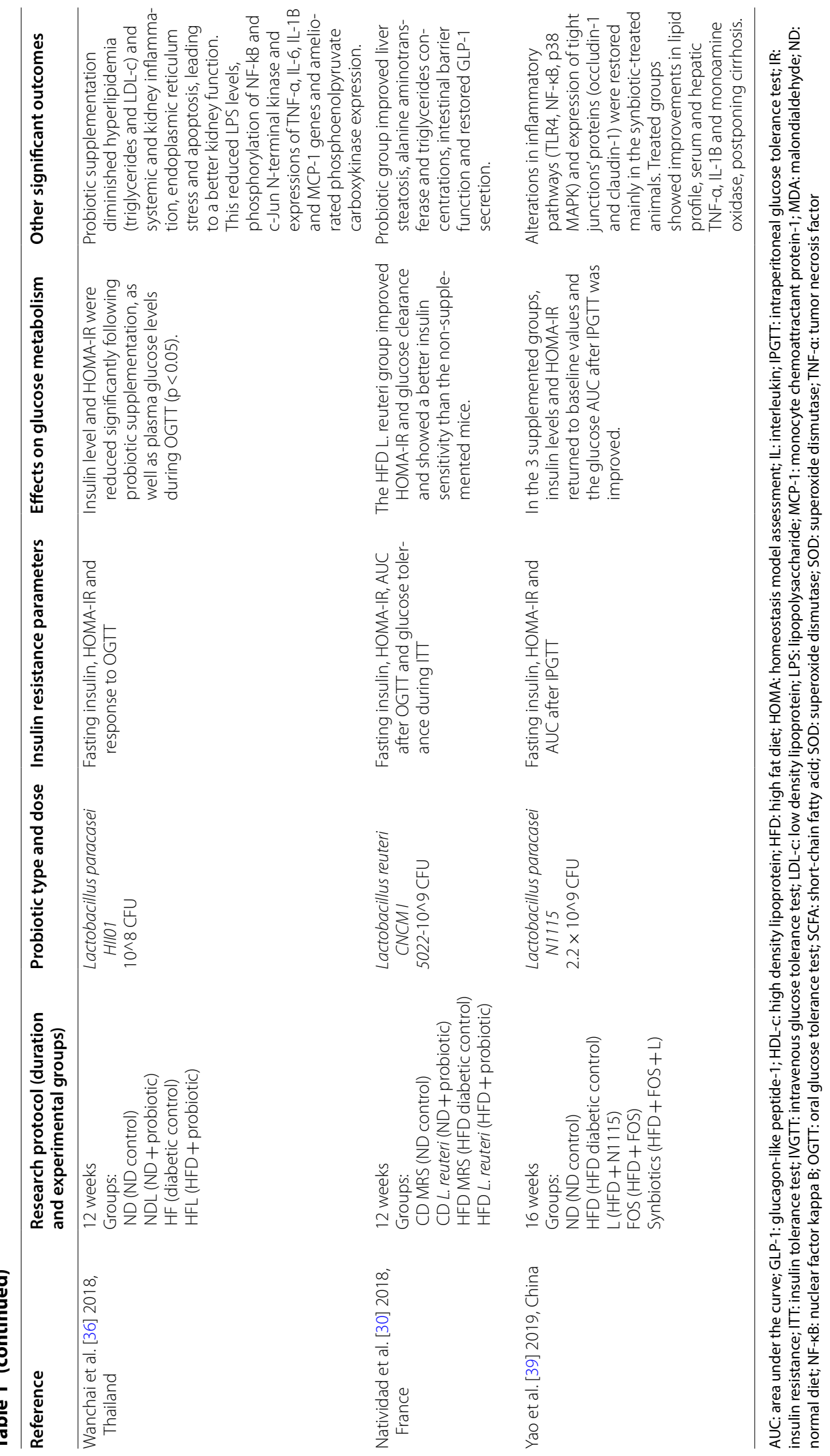


or multiple-strains probiotics, treated arms showed improvement in the glucose metabolism.

\section{Additional findings obtained with single-strain probiotics} Lactobacillus In a Swedish study of HFD-fed mice supplemented with $L$. plantarum, the lack of response only to intravenous glucose tolerance test was attributed to the administration route that was unable to stimulate glucagon-like peptide-1 (GLP-1) and insulin release [18]. L. plantarum was also used in a Korean study after having been selected from 426 strains [7]. Reduction in insulin resistance parameters was accompanied by cellular evidence of improvement in insulin signaling and expression of genes involved in inflammation.

An intervention with $L$. casei in fructose-fed hyperinsulinemic mice increased abundances of Lactobacillus and Bifidobacterium and decreased Clostridium. A lower insulinemia during oral glucose tolerance test (OGTT) was associated with GLP-2 reduction mediated by $\mathrm{Bac}$ teroides fragilis [25]. Benefits in markers of liver function and gene expressions were also observed.

Administration of $L$. coryniformis to HFD-fed mice induced vascular benefits that could be related to changes in microbiota composition and intestinal permeability with reduction in metabolic endotoxemia [28].

In a study conducted in Taiwan, lyophilized and live L. reuteri supplementations increased Lactobacillus and decreased pathogens' abundances improving intestinal barrier. Both reduced weight gain and induced improvement in lipid profile and hepatic steatosis, inflammation and gene expressions associated with insulin resistance [29]. L. reuteri was also used in a French study which evaluated the effects of its supplementation and an Aryl Hydrocarbon Receptor agonist, previously associated with MS [30]. The supplementation improved glucose metabolism and hepatic alanine transaminase levels.

In a Korean study, HFD-fed mice supplemented with L. sakei gained less weight and epididymal fat, reduced inflammation and improved the intestinal barrier (increased gene expressions of tight-junction proteins) [31].

Two studies regarding the effects of pioglitazone and different doses $L$. casei in diabetic mice showed comparable reductions in insulin resistance, TNF- $\alpha$ and IL- 6 at $10^{9} \mathrm{CFU}$ dose [16, 32]. Probiotic-induced improvement of glucose homeostasis was associated with increased abundance of Lactobacillus and Bifidobacterium and SCFA levels. Histologically, probiotic intervention prevented pancreatic islets degeneration and/or induced regeneration.

A study comparing metformin, vildagliptin and three strains of L. rhamnosus (GG, MTCC5690, MTCC5689) improved areas under the curve during insulin tolerance test and OGTT, except for the HFDMTCC5690-treated [33]. Probiotics improved intestinal permeability, increased adiponectin and reduced inflammatory markers. Metabolic benefits achieved with probiotics were similar to those obtained with metformin and vildagliptin.

An Indian study in diabetic mice found better effects in glucose metabolism, lipid profile and markers of oxidative stress with $L$. rhamnosus NCDC17 than with L. rhamnosus $G G$ supplementation [34]. The former induced reduction in inflammatory markers (TNF- $\alpha$ and IL-6) and elevation in expressions of adiponectin, proglucagon and prohormone convertase genes.

Lactobacillus rhamnosus was administered in HFD-fed mice to reduce Bilophila wadsworthia abundance which is associated with MS [21]. Supplementation prevented the $B$. wadsworthia increase in parallel to the benefits in glucose metabolism, but only under condition of high abundance of these bacteria, probiotic ameliorated the consequences of HFD.

In a Japanese study that used the strain L. gasseri in fermented milk, the supplementation increased insulin secretion by the suppression of pancreatic and systemic inflammation [35].

In a Thai study, L. paracase $i$ administered to obese rats induced benefits in glucose and lipid metabolism and in several markers of renal function and inflammation [36].

Four studies compared the effects of probiotics, prebiotics and synbiotics (combination of pro- and prebiotics). In all, Lactobacillus was used, and prebiotics were xylooligosaccharide (XOS), inulin or fructooligosaccharides (FOS). The first one evaluated the dysbiosis and inflammation in obese rats; the synbiotic group had better outcomes [8]. Treated groups decreased Firmicutes-toBacteroidetes ratio and LPS levels, but Bifidobacterium abundance increased only in the prebiotic group. Authors concluded that prebiotics, probiotics and synbiotics contributed to reduce endotoxemia and inflammation. The second examined the gut-brain axis in obese insulinresistant rats by analyzing the relationship of cognitive function and administrations of L. paracasei, XOS and their combination [37]. Prebiotic, probiotic, and synbiotic interventions improved glucose and lipid metabolism, but only XOS and synbiotic attenuated adiposity. All treated groups restored cognitive function (improved hippocampal plasticity, brain mitochondrial function and decreased microglial activation). The third study investigated association between psychiatric disorders and oxidative stress after L. plantarum supplementation, inulin or their combination [38]. All interventions improved oxidative stress with beneficial psychotropic effects (depressive and anxiety-like behaviors). In the fourth study, in which L. paracasei, FOS or the combination 
was administered to HFD-fed mice, the effects on nonalcoholic fatty liver disease (NAFLD) were evaluated [39]. Benefits were found during intraperitoneal GTT in all treated groups as well as amelioration of intestinal barrier, reduction in LPS levels and activation of insulin signaling pathways. Steatosis, dyslipidemia and inflammation were improved.

Bifidobacterium A study [40] that examined the role of sterilized $B$. longum in obese mice observed less weight gain, visceral fat accumulation and inflammation in parallel to improvement in glucose and lipid metabolism.

A comparison of B. lactis and B. longum supplementations showed better performance of the former regarding acetate levels elevation and metabolic benefits [41].

Two doses of $B$. breve had similar effects in reducing weight gain and visceral fat and in gene expressions in HFD-induced diabetic mice [19].

In an experiment with $B$. animalis, authors proposed that the probiotic could have increased beneficial commensal bacteria reducing endotoxemia and inflammation [20].

Akkermansia muciniphila Akkermansia muciniphila was administered to metabolically healthy mice showed benefits in glucose metabolism in parallel to improvement in endoplasmic reticulum stress and inflammation [26].

In a study of metformin-treated diabetic rats, different doses of live and pasteurized Akkermansia were compared [27]. Independently of the dose, probiotic had no change in glucose tolerance, in contrast to metformin that induced a lower glycemic peak during the tests. Both Akkermansia preparations reduced hepatic glycogen and inflammation markers levels.

Clostridium Supplementation with a butyrate-producing strain, $C$. butyricum, resulted in benefits in glucose and lipid metabolism that were accompanied a better intestinal barrier and anti-inflammatory effects [15].

\section{Additional findings obtained with multiple-strains probiotics} One study used a mix of Lactobacillus and Bifidobacterium [42] and another two Lactobacillus strains plus one of Bifidobacterium [43].

In HFD-induced obese mice, Lactobacillus and Bifidobacterium mixture induced favorable changes in microbiota composition, body adiposity, insulin resistance and dyslipidemia [42]. Comparing the isolated effect of each probiotic against the mixture, more pronounced protective effect of the mixture was attributed mainly to the $B$. animalis.

In a Brazilian study, a mixture of three probiotics was able to modulate microbiota composition and improved intestinal permeability, endotoxemia and inflammation [43]. The intervention was also associated with reduction in hypothalamic insulin and leptin resistance, affecting feeding behavior (reduced food intake and weight gain).

\section{Clinical trials}

Outcomes related to insulin sensitivity or resistance varied among the clinical trials as shown in Table 2. In five, significant improvement in at least one parameter [17, $22-24,44]$ was observed while in two others no benefit in insulin sensitivity was found [40, 45]. Three studies used single strains of Lactobacillus [24, 44, 46], one proof-of-concept exploratory study used single strain of Akkermansia muciniphila [23] and other three tested multi-strains (two, three and six strains containing Lactobacillus and Bifidobacterium) [17, 22, 45].

\section{Additional findings obtained with single-strain probiotic interventions}

Four single-strain interventions were found: one in healthy subjects [44], two in those with MS [23, 24] and in one with type 2 diabetes [46].

In 45 Japanese young subjects [44], a 6-week supplementation with $L$. salivarius UBL S22, combined or not with prebiotic FOS, improved inflammatory markers and lipid profile significantly in parallel to the improvement in insulin resistance. These effects were accompanied by increase in Lactobacillus abundance and decrease in reactive oxygen species production in intestinal mucosa. Performance of the synbiotic group was better than the probiotic group.

An Austrian study [24] evaluated the impact of L. casei Shirota supplementation for 12 weeks on the levels of trimethylamine $\mathrm{N}$-oxide (TMAO) in 30 subjects with MS. Insulin sensitivity index improved but not other parameters such as HOMA-IR, quantitative insulin sensitivity check index (QUICKI) and Matsuda. No benefits were found in lipid or inflammation markers. TMAO levels decreased significantly in both groups and did not differ between them.

A Taiwanese placebo-controlled clinical trial of 68 type 2 DM subjects treated with a single strain of $L$. reuteri ADR-1 or ADR-3 [46], no change in insulin sensitivity was observed, but the L. reuteri ADR-1-treated group showed reductions in glycated hemoglobin (HbA1c), cholesterol, and antioxidants proteins (GPX and SOD), while the ADR-3-treated group had blood pressure and IL- $1 \beta$ reduced.

A small clinical trial with $A$. muciniphila, published as proof-of-concept [23], showed that live or pasteurized probiotics in high doses were safe and well tolerated. Treatments restored gut microbiota and barrier function and improved several metabolic parameters. Both 


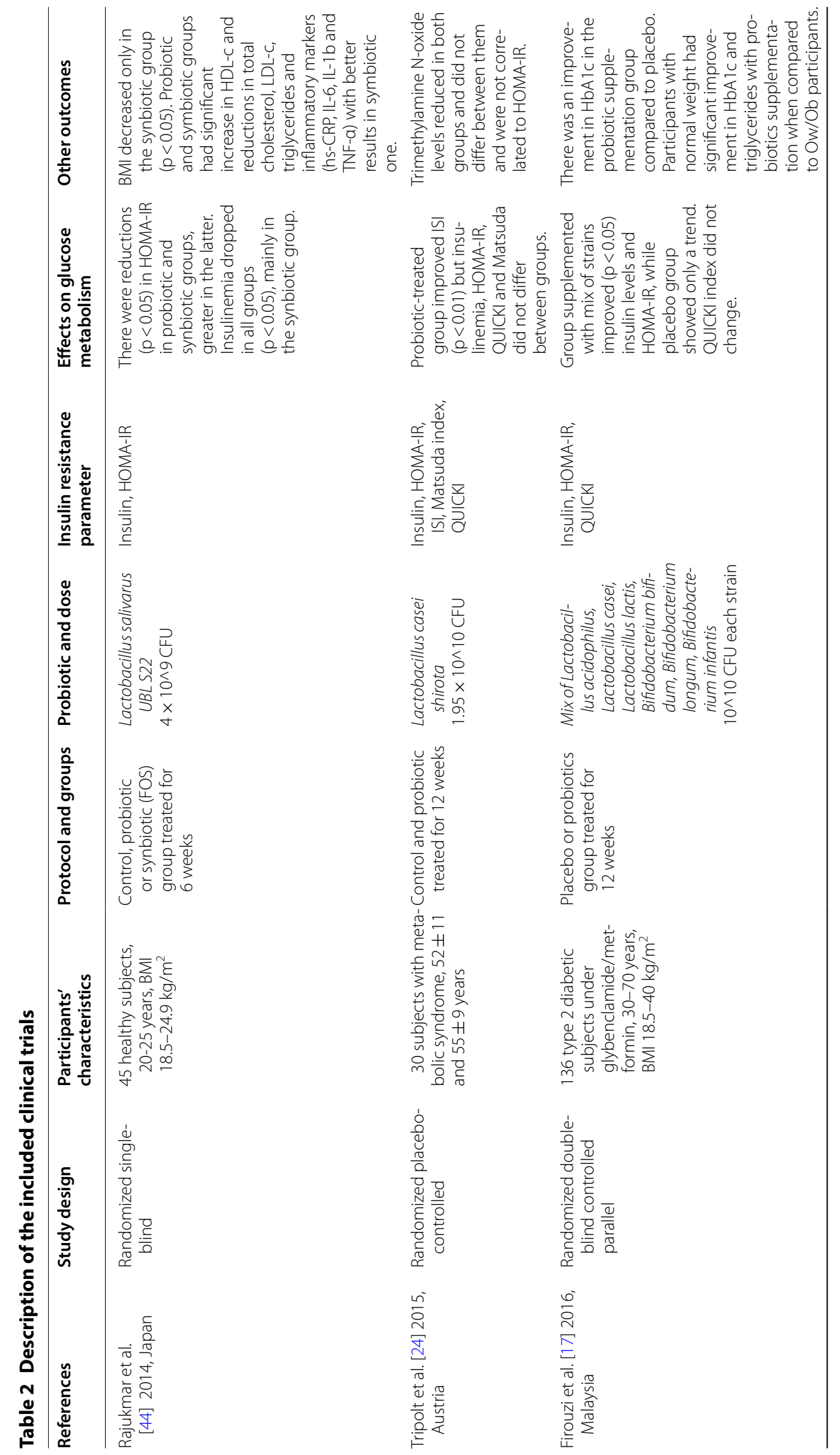




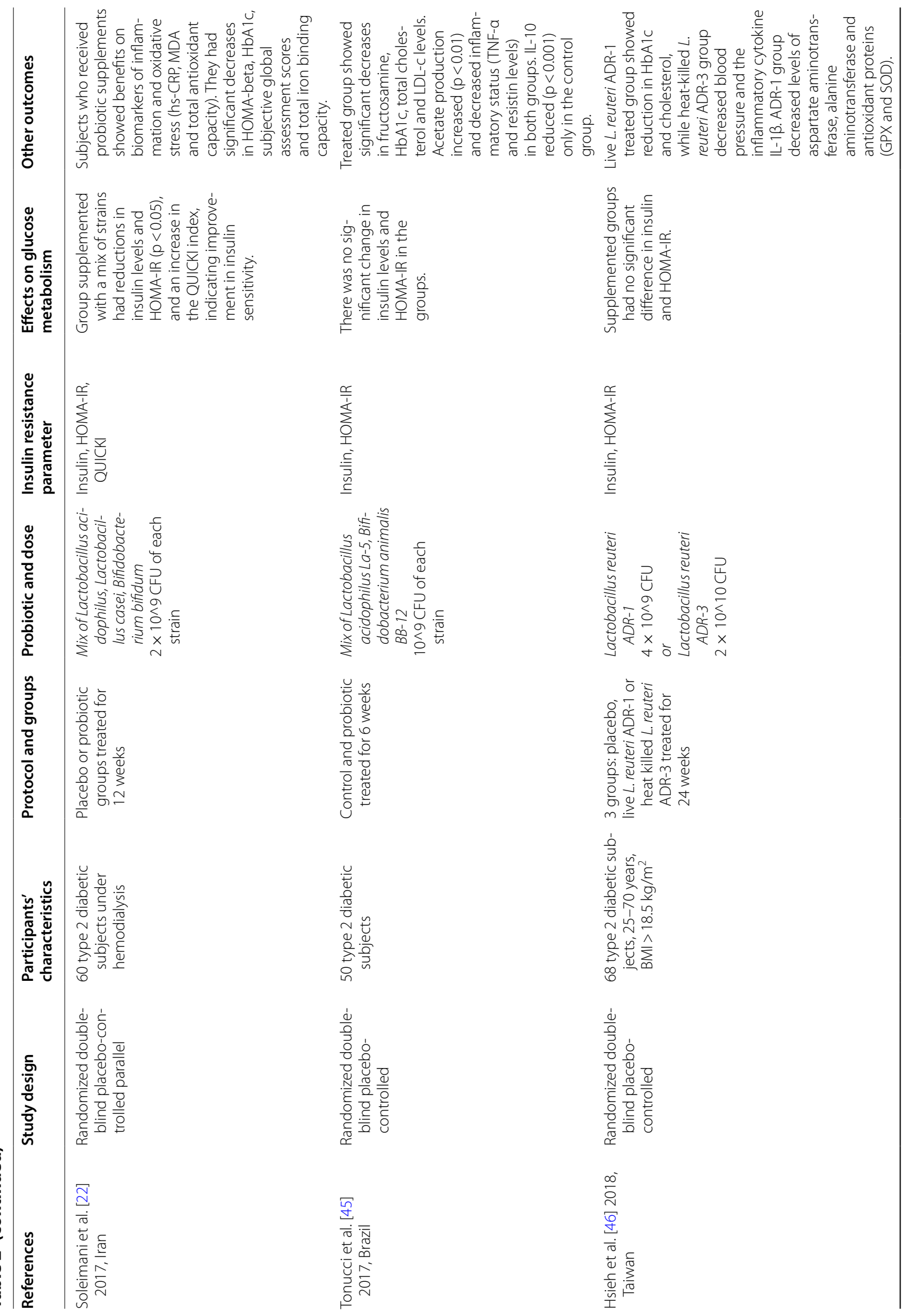




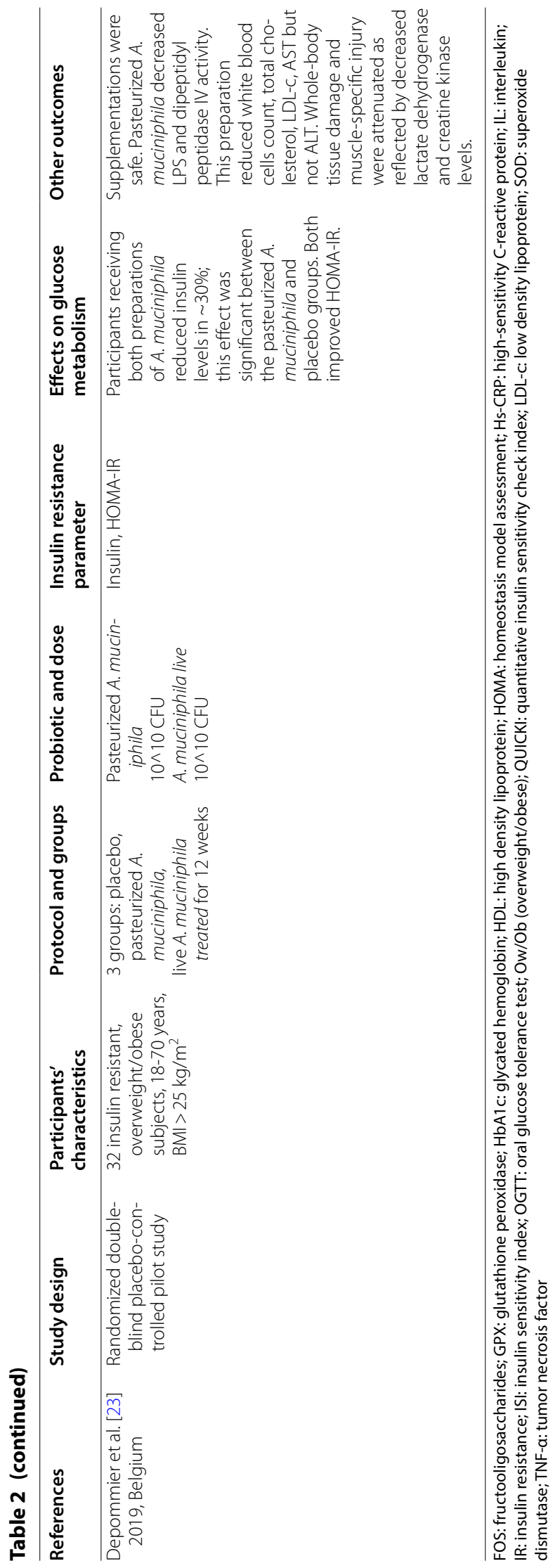


preparations were effective but pasteurized had a better performance.

\section{Additional findings obtained with multiple-strains probiotic interventions}

In a Brazilian placebo-controlled study, individuals under antidiabetic treatment who consumed fermented goat milk containing $L$. acidophilus and B. animalis did not change insulin resistance parameters but $\mathrm{HbA1c}$, lipids and inflammatory markers were improved [45].

In a Malaysian placebo-controlled trial of diabetic individuals, HOMA-IR and HbA1c was improved by multiple-strains of probiotic intervention [17].

In an Iranian controlled study, diabetic individuals on hemodialysis treated with multiple strains of Lactobacillus also had $\mathrm{HbA1c}$ reduction in addition to improvement in insulin sensitivity indexes [22].

\section{Discussion}

This systematic review is the first to focus on the role of probiotics in attenuating insulin resistance, involved in prevalent diseases of the contemporary world. Body of evidence was mostly based on experiments conducted in animals that supported benefits of certain supplements in attenuating induced states of insulin resistance. Underlying mechanisms and markers of improvement in insulin signaling were consistently demonstrated. In humans, existing literature are limited, and heterogeneity of study designs and samples allowed collecting reliable findings but impeded meta-analysis. Actually, most studies have been published in the last 5 years, motivated by the growing knowledge on the importance of the gut microbiome for cardiometabolic health. Despite promising results as an adjuvant therapeutic modality for controlling disorders linked by insulin resistance, there is still insufficient data in humans to support widespread probiotic prescription.

\section{Probiotic, its dose and duration of interventions}

Most used probiotics in research and clinical settings were species of Lactobacillus whose benefits for health have been demonstrated [5, 44, 47]. These commensal gram-positive bacteria belong to phylum Firmicutes. Beneficial effects to the gastrointestinal tract, immune system, metabolism and anticarcinogenic properties have been reported $[4,5,9]$. Bifidobacterium (phylum Actinobacteria) are anaerobic and, as Lactobacillus, have been added to fermented products. Optimal doses of these probiotics were already known $[4,9]$ and even sterilized bacteria have been considered functional [27, 29, 40]. Certain butyrate-producing species of Clostridium (phylum Firmicutes) have been associated with metabolic benefits, as well as the $A$. muciniphila, a mucin-degrading bacterium (phylum Verrucomicrobia). In contrast to the other probiotics, literature about Akkermansia is not abundant; safety and tolerability of live and pasteurized A. muciniphila were already investigated [23]. Researchers have used $A$. muciniphila as a pasteurized probiotic $[27,29,40]$ because the pasteurization process potentializes its effects in the host $[23,48]$.

Some authors compared effects of probiotics and synbiotics in animals and both strategies resulted in benefits to intestinal barrier function and restored immune response [15]. This is partially in agreement with a trial in which a better performance was detected for the synbiotic group [44]. Explanation was based on the fact that acetate and lactate produced by Lactobacillus and Bifidobacterium can be converted to butyrate by other intestinal bacteria.

Most studies worked with probiotic doses from $10^{8}$ to $10^{10} \mathrm{CFU} /$ day in both human and animal. It is necessary to maintain a content higher than $10^{6} \mathrm{CFU}$ in the intestine to play an appropriate probiotic role [16]. In all studies reviewed except one [27], any dose in that range administered resulted in significant improvement in insulin resistance $[16,40]$ and doses higher than $10^{10}$ had no additional benefit [16]. Regarding live $A$. muciniphila, experiments in animals suggested that it may be required low doses and an interval between them since, in excess, could exacerbate its mucolytic function and can be harmful to the intestinal mucosa [27]. However, preliminary results obtained in a recent clinical trial, a $10^{10} \mathrm{CFU}$ dose showed to be well tolerated and safe [23].

The duration of probiotic administration seemed crucial for the intervention efficacy. 12-week compared to 6-week interventions showed better results especially in animals but also in humans. Interestingly, in human studies, benefits in certain parameters of glucose metabolism and inflammatory markers were found although improved insulin sensitivity was not confirmed. We speculate that 12 week-intervention may be insufficient to get an impact on insulin sensitivity and longer trials should be further conducted. Also unknown is how persistent is the effect of the probiotic therapy when it ceased. Possibly, the probiotic-induced changes in microbiota are not permanent and its composition will return to be driven by dietary habits. Even fecal transplantation that has shown promising results in humans with obesity and SM cannot be considered as a definitive therapy for insulin resistance $[9,49]$.

\section{Probiotics and insulin resistance in animal studies}

All animal studies probiotic interventions reviewed had control groups and their results were appropriately compared. The vast majority used HFD as a strategy for inducing weight gain, insulin resistance and impaired 
glucose tolerance, and rarely fructose or streptozotocin. This has been considered an adequate model to mimic the impact of Western diet in humans. There is consistent evidence of gut microbiota changes caused by high saturated fat intake, but controversial findings related to Firmicutes/Bacteroidetes ratio were reported, with increased [8], neutral [41] and even decreased [43] results among the studies. This dysbiosis elevates LPS content and compromises intestinal mucosal integrity. Locally, LPS reduces expression of tight junctions' proteins impairing the barrier function. LPS translocation from the lumen into circulation can trigger metabolic endotoxemia; its binding to TLR- 4 activates transcription of NF-kB and production of inflammatory cytokines that deteriorate insulin signaling. Therefore, a state of systemic low-grade inflammation and insulin resistance may be resultant from an inadequate diet. In the articles reviewed, HFD or fructose-enriched diet were effective to induce weight gain, systemic inflammation and a variety of metabolic abnormalities.

As the main outcome of the present review was insulin resistance or insulin sensitivity at least one parameter was reported. All except one animal study used indirect methods to assess insulin sensitivity. The gold standard method, euglycemic hyperinsulinemic clamp, was employed only in animal models in a Brazilian study by Baggaroli et al. [43]. Other investigators used indirect methods: HOMA-IR [7, 8, 16, 19, 21, 28-30, 32, 33, 36, $37,39,42]$, areas under the curve obtained by OGTT [ 7 , $8,15,16,18,20,21,25,27-37,40,41]$, intravenous [18] and intraperitoneal GTT [20, 26, 39, 42, 43], ITT [7, 15, $30,32,33,40]$, which are reliable to estimate insulin sensitivity and contributed for interpretations of the probiotics' effects.

Several studies, based on diverse probiotic preparations, reported their findings in different organs, also at molecular level and changes in gene expressions $[16,19$, $25,29,32-34,36,38,47$ ] involved in underlying mechanisms of oxidative stress, inflammation and insulin resistance. HFD-induced mice showed worsening in proinflammatory cytokines and claudin and occludin gene expressions in the intestinal wall which ameliorated after probiotics interventions [33, 39]. A study [15] included in the present review observed that HFD-fed mice treated with $C$. butyricum increased tight-junction proteins' expression in parallel to LPS levels reduction. Additionally, intervention with this Clostridium increased SCFA, improved intestinal permeability, insulin resistance and hepatic steatosis [15].

As a matter of fact, local and systemic benefits were described in association with SCFA produced by specific bacteria $[5,6,50]$. It is known that HFD-fed obese as well as diabetic mice have reduced SCFA levels and that fermented foods containing probiotics have the ability to increase these metabolites in the colon [32]. Butyrate exerts important roles in the intestinal immune system $[6,50]$ and in inhibition of the NF-kB in colonocytes [15], favoring an anti-inflammatory condition. Acetate promotes GLP-1 secretion by L cells, modulating glucose metabolism, central regulation of appetite and reducing adipocytes hypertrophy $[41,51]$. Studies which employed SCFA-producing bacteria as Clostridium [15], Akkermansia $[26,27]$ and their metabolites, may have contributed to improvements of insulin resistance parameters.

Benefits of metabolic profile from probiotics consumption by rodents supports that they occurred at least in part via changes in the microbiota composition and intestinal barrier with systemic repercussions. Such effects were described in $96.3 \%$ of the animal studies with species of Lactobacillus [7, 8, 16, 18, 21, 25, 28-39, 42, 43] and Bifidobacterium [19, 20, 40-43], the C. butyricum [15] and A. muciniphila [26] while the supplementation lasted. Considering that their beneficial effects are desirable to control cardiometabolic diseases, deepen knowledge about long-term interventions and its outcomes is needed. Animal models studied were insulinresistant which confers risk to atherosclerosis. A recent atherosclerosis marker, TMAO [24], is dependent on choline and carnitine metabolism by intestinal bacteria action. Probiotics have been proposed as a new therapeutic target against cardiovascular disease [28, 52]. In fact, diabetic mice treated with $L$. plantarum or prebiotic FOS reverted dysbiosis, attenuated inflammatory proteins' expression and reduction of cardiovascular risk [52]. However, L. casei Shirota supplementation in humans showed no difference in TMAO levels when compared to untreated subjects although contrasting findings were found in animal studies [24].

Different research protocols among the reviewed studies impeded comparisons of probiotic efficacy among them and none investigated whether there would be a residual effect after suspending probiotic administration. Anyhow, these promising results of animal studies have motivated studies in humans considering that prevalent lifestyle-related diseases are triggered by insulin resistance.

\section{Probiotics and insulin resistance in clinical trials}

There is mounting evidence pointing out to the role of gut dysbiosis in the pathogenesis of metabolic disorders, such as obesity, type 2 DM, MS and NAFLD [47]. Since studies demonstrated that reduction in gut microbiome diversity and richness in obese subjects were associated with high risk for insulin resistance-linked diseases [47], this ecosystem emerged as a target to improve lifestylerelated metabolic disturbances [53]. 
Our review highlights the scarcity and heterogeneity of human studies which tested the efficacy of probiotic supplementation in attenuating insulin resistance and reducing its cardiometabolic consequences. Sample characteristics and intervention design (probiotic type, dose and duration) differed among the studies reviewed. Four studies used single strains for 6, 12 and 24 weeks, in healthy young subjects [44] or with MS [23, 24] or type 2 DM [46], respectively, while others used multi strains blends for 6 [45] or 12 weeks [17, 22]. Also, different assessment of insulin sensitivity/resistance was employed; all trials used at least insulinemia and HOMAIR as parameters of insulin resistance and none of the clinical trials used the gold standard technique. Investigators also used other indirect methods like insulin sensitivity index (ISI) [24], Matsuda [24] and QUICKI $[17,22,24]$ to estimate the probiotics' effects on insulin sensitivity. In the longest intervention (24 weeks) including diabetic subjects, no change in insulin resistance was observed [46], but in shorter ones [17, 22]. Subjects with MS treated with Lactobacillus [24] improved the ISI without changing HOMA-IR, in contrast to the intervention with A. muciniphila [23] that reduced HOMA-IR. In healthy subjects [44], 6 week-supplementation was sufficient to reduce insulinemia and HOMA-IR. Facing such methodological heterogeneity, no meta-analysis was available in literature.

Articles reviewed suggested probiotics use as preventive or adjuvant therapy for metabolic diseases, such as obesity and type 2 DM $[44,46]$. In general, when excessive body weight was present probiotic supplementation appeared to be more effective than in eutrophic individuals [17], but conflicting findings were found among the trials $[17,22,24,44]$. We hypothesized that normalweight subjects on a healthy diet should already have a balanced microbiota composition, an adequate intestinal barrier and a normal immune system functioning. However, even in healthy young eutrophic subjects, a Japanese study obtained improvement in several metabolic parameters with probiotic supplementation.

The Bacteroidetes-to-Firmicutes ratio has been used to reflect the association of gut microbiota with body adiposity also in humans [54]. Increased ratios were reported in obese individuals or in populations consuming obesogenic diets [9], although results were not consistent $[17,46]$. Weight loss in obese subjects was able to change microbiota composition without altering proportions of Bacteroidetes and Firmicutes [55]. Crosstalk between intestinal bacteria and host is relevant to determine the inflammatory state and glucose tolerance $[26,53,56]$. In fact, the role of the cytokine IL-22 in maintaining integrity of the intestinal barrier with attenuated metabolic disorders was demonstrated [57].
In the trials reviewed, such possible benefit of probiotics' supplementation was not investigated. Association of SCFA-producing bacteria and decrease in inflammation and insulin resistance was previously reported in animals and humans [5, 6, 9]. Particularly, a role for butyrate was described in the prevention of obesity by regulating incretins and anorexigenic hormones production (GLP-1 and peptide $Y Y$ ), appetite and energy expenditure [47]. In our review, SCFA-producing bacteria supplemented in humans used were A. muciniphila and strains of Lactobacillus and Bifidobacterium [17, 22-24, 44-46].

Protective effects of the administration of A. muciniphila in intestinal barrier and metabolic disorders have been mostly investigated in animals [26, 27]. More recently, growing evidence has indicated potential benefits in individuals with obesity and type $2 \mathrm{DM}[23,58]$. The first randomized, double-blind, placebo-controlled trial, using live or pasteurized A. muciniphila, in a small sample of insulin-resistant individuals, looked at safety and tolerability but also at metabolic parameters. Improvement in insulin sensitivity and other parameters have motivated several ongoing trials.

\section{Probiotics compared to antidiabetic agents}

Interest of the scientific community has grown regarding the application of probiotics as an adjuvant therapy for insulin resistance syndromes. Currently, there are some indications of certain strains for preventive or adjuvant therapeutic purposes $[4,6,9,45]$. Although probiotics in combination with drugs could be valuable in the treatment of prediabetes and type $2 \mathrm{DM}$, the studies reviewed analysis does not assure the efficacy of their isolated use. As insulin resistance is part of the natural history of cardiometabolic diseases, established interventions (lifestyle changes and medications) should be employed. The use of probiotics by at-risk individuals, together with behavioral measures (diet and physical activity), seemed to be useful according to preliminary results, although require confirmation in further studies.

Three animal studies that compared probiotics (L. casei CCFM449, L. rhamnosus GG, MTCC5689, MTCC5689) to antidiabetic agents (pioglitazone, vildagliptin and metformin) found similar significant improvement in insulin resistance and inflammatory markers $[16,32,33]$. In contrast, the combination of probiotics and/or prebiotics with antidiabetic drugs (metformin and sitagliptin) improved the incretin effect and insulin sensitivity in mice [59]. Although vildagliptin acts directly in GLP-1 regulation and metformin also contribute to increase its levels, similar effects were identified for probiotics suggesting that regulation of GLP-1 is a possible mechanism which favors glucose homeostasis $[60,61]$. These findings support that probiotics combined with antidiabetic 
agents may be promising in the treatment of glucose metabolism disturbances. Further studies in humans are necessary to test this hypothesis.

The scenario disclosed by the present review shows that animal studies are still important to fill gaps on the role and mechanisms by which probiotics can enhance insulin sensitivity. Also, it has been encouraging to develop clinical trials in strata of populations, using standardized experimental conditions. Not only the animal intestinal microbiome differs from humans, but there are differences among populations worldwide [24]. In addition to lifestyle, other environmental conditions, use of antibiotics and genetic factors should influence probiotics response [4]. Advances in the knowledge on metabolic effects of probiotics, prebiotics and/or synbiotics for ameliorating microbiota composition and insulin resistance could optimize outcomes of fecal transplantation in humans with MS.

Despite the amplitude of this review, the main limitation was related to heterogeneity of samples and research protocols involving a variety of probiotics used alone or in combinations. A strength of this publication is that despite having few publications, this review collected both animal and human studies. As possible effects of supplementation with multiple strains combined are unclear, we alert that inter-strain competition and even deleterious changes in microbiota composition might occur. Standardized methods that enable the comparison of different studies and the elaboration of meta-analysis were highly warranted.

In summary, this review concluded that available data regarding the effects of certain probiotics do not guarantee sustained amelioration of insulin resistance in humans. Consistent beneficial results for intestinal barrier function, immune system and metabolism were reported in animal models, which may encourage longterm randomized clinical trials in people with obesity and at cardiometabolic risk. It is possible that supplementation with probiotics in combination with medications and/or prebiotics, combined with a healthy lifestyle, will prove useful in the current world scenario. Further research is necessary to test hypothesis raised in the present review.

\section{Supplementary information}

Supplementary information accompanies this paper at https://doi. org/10.1186/s13098-020-00603-6.

Additional file 1. Detailed procedures for the systematic review including its search equations.

\section{Abbreviations}

DM: Diabetes mellitus; FOS: Fructooligosaccharides; GLP: Glucagon-like peptide; GPX: Glutathione peroxidase; GTT: Glucose tolerance test; HbA1c:
Glycated hemoglobin; HFD: High fat diet; HOMA-IR: Homeostasis model assessment-Insulin resistance; IL: Interleukin; ISI: Insulin sensitivity index; ITT: Insulin tolerance test; IVGTT: Intravenous glucose tolerance test; LPS: Lipopolysaccharide; MS: Metabolic syndrome; NAFLD: Non-alcoholic fatty liver disease; NF-kB: Nuclear factor kappa B; OGTT: Oral glucose tolerance test; QUICKI: Quantitative insulin sensitivity check index; SCFA: Short-chain fatty acid; SOD: Superoxide dismutase; TMAO: Trimethylamine N-oxide; TNF-a: Tumor Necrosis Factor; XOS: Xylooligosaccharides.

\section{Authors' contributions}

BIMS and DC participated in conceptualization, methodology, analysis and writing the manuscript. SRGF participated in conceptualization, methodology, validation and supervision of the analysis, review the writing and editing the manuscript. All authors read and approved the final manuscript.

\section{Competing interests}

The authors declare that they have no competing interests.

Received: 1 August 2020 Accepted: 20 October 2020

Published online: 11 November 2020

\section{References}

1. GBD 2015 Obesity Collaborators. Health effects of overweight and obesity in 195 countries over 25 years. N Engl J Med. 2017;377:13-27.

2. Bray G, Kim K, Wilding J, Federation WO. Obesity: a chronic relapsing progressive disease process. A position statement of the World Obesity Federation. Obes Rev. 2017;18:715-23.

3. International Diabetes Federation. IDF diabetes atlas 2019. 9th ed. Brussels: IDF; 2019.

4. Panwar H, Rashmi HM, Batish VK, Grover S. Probiotics as potential biotherapeutics in the management of type 2 diabetes - prospects and perspectives. Diabetes Metab Res Rev. 2013;29(2):103-12.

5. Tonucci LB, Santos KMO, Ferreira CLLF, Ribeiro SMR, Oliveira LL, Martino HSD. Gut microbiota and probiotics: focus on diabetes mellitus. Crit Rev Food Sci Nutr. 2015;57(11):2296-309.

6. Barz ML, Anhê FF, Varin TV, Desjardins Y, Levy E, Roy D, et al. Probiotics as complementary treatment for metabolic disorders. Diabetes Metab J. 2015:39(4):291-303.

7. Lee E, Jung SR, Lee SY, Lee NK, Paik HD, Lim SI. Lactobacillus plantarum strain Ln4 attenuates diet-induced obesity, insulin resistance, and changes in hepatic mRNA levels associated with glucose and lipid metabolism. Nutrients. 2018;10(5):643-58.

8. Thiennimitr P, Yasom S, Tunapong W, Chunchai T, Wanchai K, Pongchaidecha A, et al. Lactobacillus paracasei HII01, xylooligosaccharides, and synbiotics reduce gut disturbance in obese rats. Nutrition. 2018;54:40-7.

9. Hampe CS, Roth CL. Probiotic strains and mechanistic insights for the treatment of type 2 diabetes. Endocrine. 2017;58(2):207-27.

10. Yan X, Feng B, Li P, Tang Z, Wang L. Microflora disturbance during progression of glucose Intolerance and effect of sitagliptin: an animal study. J Diabetes Res. 2016;2016:1-10.

11. Kulecka M, et al. Prolonged transfer of feces from the lean mice modulates gut microbiota in obese mice. Nutr Metab. 2016;13:57.

12. Arumugam M, Raes J, Pelletier E, Le Paslier D, Yamada T, Mende $D R$, et al. Enterotypes of the human gut microbiome. Nature. 2011:473(7346):174-80

13. Wu GD, Chen J, Hoffmann C, Bittinger K, Chen YY, Keilbaugh SA, et al. Linking long-term dietary patterns with gut microbial enterotypes. Science. 2011;334(6052):105-8

14. Moraes ACF, Silva IT, Almeida-Pititto B, Ferreira SRG. Microbiota intestinal e risco cardiometabólico: mecanismos e modulação dietética. Arq Bras Endocrinol Metab. 2014;58(4):317-27.

15. Shang H, Sun J, Chen YQ. Clostridium butyricum CGMCC0313.1 modulates lipid rofile, insulin resistance and colon homeostasis in obese mice. PLoS ONE. 2016:11(4):1-15

16. Wang G, Li X, Zhao J, Zhang H, Chen W. Lactobacillus casei CCFM419 attenuates type 2 diabetes via a gut microbiota dependent mechanism. Food Funct. 2017;8(9):3155-64.

17. Firouzi S, Majid HA, Ismail A, Kamaruddin NA, Barakatun-Nisak MY. Effect of multi-strain probiotics (multi-strain microbial cell preparation) 
on glycemic control and other diabetes-related outcomes in people with type 2 diabetes: a randomized controlled trial. Eur J Nutr. 2016;56(4):1535-50.

18. Andersson U, Bränning C, Ahrné S, Molin G, Alenfall J, Onning G, et al. Probiotics lower plasma glucose in the high-fat fed C57BL/6J mouse. Benef Microbes. 2010;1(2):189-96.

19. Kondo S, Xiao JZ, Satoh T, Odamaki T, Takahashi S, Sugahara H, et al. Antiobesity effects of bifidobacterium breve strain B-3 supplementation in a mouse model with high-fat diet-induced obesity. Biosci Biotechnol Biochem. 2010;74(8):1656-61.

20. Amar J, Chabo C, Waget A, Klopp P, Vachoux C, Bermúdez-Humarán LG, et al. Intestinal mucosal adherence and translocation of commensal bacteria at the early onset of type 2 diabetes: molecular mechanisms and probiotic treatment. EMBO Mol Med. 2011;3(9):559-72.

21. Natividad JM, Lamas B, Pham HP, Michel ML, Rainteau D, Bridonneau C, et al. Bilophila wadsworthia aggravates high fat diet induced metabolic dysfunctions in mice. Nat Commun. 2018;9(1):1-15

22. Soleimani A, Mojarrad MZ, Bahmani F, Taghizadeh M, Ramezani M Tajabadi-Ebrahimi M, et al. Probiotic supplementation in diabetic hemodialysis patients has beneficial metabolic effects. Kidney Int. 2017;91(2):435-42.

23. Depommier C, Everard A, Druart C, Plovier H, Hul MV, Vieira-Silva S, et al. Supplementation with Akkermansia muciniphila in overweight and obese human volunteers: a proof-of-concept exploratory study. Nat Med. 2019;25(7):1096-103.

24. Tripolt NJ, Leber B, Triebl A, Köfeler H, Stadlbauer V, Sourij H. Effect of Lactobacillus casei Shirota supplementation on trimethylamine$\mathrm{N}$-oxide levels in patients with metabolic syndrome: an open-label, randomized study. Atherosclerosis. 2015;242(1):141-4.

25. Zhang Y, Wang L, Zhang J, Li Y, He Q, Li H, et al. Probiotic Lactobacillus casei Zhang ameliorates high-fructose-induced impaired glucose tolerance in hyperinsulinemia rats. Eur J Nutr. 2013;53(1):221-32.

26. Zhao S, Liu W, Wang J, Shi J, Sun Y, Wang W, et al. Akkermansia muciniphila improves metabolic profiles by reducing inflammation in chow diet-fed mice. J Mol Endocrinol. 2017;58(1):1-14.

27. Zhang L, Qin Q, Liu M, Zhang X, He F, Wang G. Akkermansia muciniphila can reduce the damage of gluco/lipotoxicity, oxidative stress and inflammation, and normalize intestine microbiota in streptozotocininduced diabetic rats. Pathog Disease. 2018;76(4):1-15.

28. Toral M, Gómez-Guzmán M, Jiménez R, Romero M, Sánchez M, Utrilla MP, et al. The probiotic Lactobacillus coryniformis CECT5711 reduces the vascular pro-oxidant and pro-inflammatory status in obese mice. Clin Sci. 2014;127(1):33-45.

29. Hsieh FC, Lan CC, Huang TY, Chen KW, Chai CY, Chen WT, et al. Heatkilled and live Lactobacillus reuteri GMNL-263 exhibit similar effects on improving metabolic functions in high-fat diet-induced obese rats. Food Funct. 2016;7(5):2374-88.

30. Natividad JM, et al. Impaired aryl hydrocarbon receptor ligand production by the gut microbiota Is a key factor in metabolic syndrome. Cell Metab. 2018;28:737-49.

31. Lim SM, Jeong JJ, Woo KH, Han MJ, Kim DH. Lactobacillus sakei OK67 ameliorates high-fat diet-induced blood glucose intolerance and obesity in mice by inhibiting gut microbiota lipopolysaccharide production and inducing colon tight junction protein expression. Nutr Res. 2016:36(4):337-48

32. Li X, Wang E, Yin B, Fang D, Chen P, Wang G, et al. Effects of Lactobacillus casei CCFM419 on insulin resistance and gut microbiota in type 2 diabetic mice. Benef Microbes. 2017;8(3):421-32.

33. Balakumar M, Prabhu D, Sathishkumar C, Prabu P, Rokana N, Kumar R, et al. Improvement in glucose tolerance and insulin sensitivity by probiotic strains of Indian gut origin in high-fat diet-fed C57BL/6J mice. Eur J Nutr. 2016;57(1):279-95

34. Singh S, Sharma RK, Malhotra S, Pothuraju R, Shandilya UK. Lactobacillus rhamnosus NCDC17 ameliorates type-2 diabetes by improving gut function, oxidative stress and inflammation in high-fat-diet fed and streptozotocin treated rats. Beneficial Microbes. 2017:8(2):243-55.

35. Niibo M, et al. Probiotic Lactobacillus gasseri SBT2055 improves insulin secretion in a diabetic rat model. J Dairy Sci. 2019;102(2):1-10.

36. Wanchai $\mathrm{K}$, et al. Probiotic Lactobacillus paracasei HII01 protects rats against obese-insulin resistance induced kidney injury and impaired renal organic anion transporter (Oat3) function. Clin Sci. 2018;132(14):1545-63.

37. Chunchai $T$, et al. Decreased microglial activation through gutbrain axis by prebiotics, probiotics, or synbiotics effectively restored cognitive function in obese-insulin resistant rats. J Neuroinflamm. 2018;15(1):11.

38. Morshedi M, et al. Beneficial psychological effects of novel psychobiotics in diabetic rats: the interaction among the gut, blood, and amygdala. J Nutr Biochem. 2018;57:145-52.

39. Yao F, et al. Effect of Lactobacillus paracasei N1115 and fructooligosaccharides in nonalcoholic fatty liver disease. Arch Med Sci. 2019;15(5):1336-44.

40. Kikuchi K, Othman MB, Sakamoto K. Sterilized bifidobacteria suppressed fat accumulation and blood glucose level. Bioch Biophys Res Commun. 2018;501(4):1041-7.

41. Aoki R, Kamikado K, Suda W, Takii H, Mikami Y, Suganuma N, et al. A proliferative probiotic Bifidobacterium strain in the gut ameliorates progression of metabolic disorders via microbiota modulation and acetate elevation. Sci Rep. 2017;7:43522.

42. Alard J, Lehrter V, Rhimi M, Mangin I, Peucelle V, Abraham AL, et al. Beneficial metabolic effects of selected probiotics on diet-induced obesity and insulin resistance in mice are associated with improvement of dysbiotic gut microbiota. Environ Microbiol. 2016;18(5):1484-97.

43. Bagarolli RA, Tobar N, Oliveira AG, Araújo TG, Carvalho BM, Rocha GZ, et al. Probiotics modulate gut microbiota and improve insulin sensitivity in DIO mice. J Nutr Biochem. 2017;50:16-25.

44. Rajkumar H, Kumar M, Das N, Kumar SN, Challa HR, Nagpal R. Effect of probiotic Lactobacillus salivarius UBL S22 and prebiotic fructo-oligosaccharide on serum lipids, inflammatory markers, insulin sensitivity, and gut bacteria in healthy young volunteers. J Cardiovasc Pharmacol Ther. 2014;20(3):289-98.

45. Tonucci LB, Santos KMO, Oliveira LL, Ribeiro SMR, Martino HSD. Clinical application of probiotics in type 2 diabetes mellitus: a randomized, double-blind, placebo-controlled study. Clin Nutr. 2017;36(1):85-92.

46. Hsieh MC, et al. The beneficial effects of Lactobacillus reuteri ADR-1 or ADR-3 consumption on type 2 diabetes mellitus: a randomized, double blinded, placebo-controlled trial. Sci Rep. 2018;8:16791.

47. Vallianou N, et al. Probiotics, prebiotics, synbiotics, postbiotics, and obesity: current evidence, controversies, and perspectives. Curr Obes Rep. 2020;9(3):179-92.

48. Plovier H, Everard A, Druart C, Depommier C, Hul MV, Geurts L, et al. A purified membrane protein from Akkermansia muciniphila or the pasteurized bacterium improves metabolism in obese and diabetic mice. Nat Med. 2016;23(1):107-13.

49. Zhang Z, Mocanu V, Cai C, Dang J, Slater L, Deehan EC, et al. Impact of fecal microbiota transplantation on obesity and metabolic syndrome-a systematic review. Nutrients. 2019;11(10):2291-307.

50. Lin HV, Frassetto A, Kowalik EJJ, Nawrocki AR, Lu MM, Kosinski JR, et al. Butyrate and propionate protect against diet induced obesity and regulate gut hormones via free fatty acid. PLoS ONE. 2012;7(4):e35240.

51. Frost G, Sleeth ML, Sahuri-Arisoylu M, Lizarbe B, Cerdan S, Brody L, et al. The short-chain fatty acid acetate reduces appetite via a central homeostatic mechanism. Nat Commun. 2014:5(1):3611.

52. Liu WC, Yang MC, Wu YY, Chen PH, Hsu CM, Chen LW. Lactobacillus plantarum reverse diabetes-induced $\mathrm{Fmo3}$ and ICAM expression in mice through enteric dysbiosis-related c-Jun NH2-terminal kinase pathways. PLOS ONE. 2018;13(5):1-23.

53. Cani PD, Bibiloni R, Knauf C, Waget A, Neyrinck AM, Delzenne NM, et al. Changes in gut microbiota control metabolic endotoxemia-induced inflammation in high-fat diet-induced obesity and diabetes in mice. Diabetes. 2008;57(6):1470-81.

54. Ley R, et al. Human gut microbes associated with obesity. Nature. 2006;444:1022-3.

55. Duncan SH, Lobley GE, Holtrop G, et al. Human colonic microbiota associated with diet, obesity and weight loss. Int J Obes. 2008:32(11):1720-4.

56. Cani PD, Amar J, Iglesias MA, Poggi M, Knauf C, Bastelica D, et al. Metabolic endotoxemia initiates obesity and insulin resistance. Diabetes. 2007;56(7):1761-72. 
57. Wang X, Ota N, Manzanillo P, Kates L, Zavala-Solorio J, Eidenschenk C, et al. Interleukin-22 alleviates metabolic disorders and restores mucosal immunity in diabetes. Nature. 2014;514(7521):237-41.

58. Dao MC, et al. Akkermansia muciniphila and improved metabolic health during a dietary intervention in obesity: relationship with gut microbiome richness and ecology. Gut. 2015;65(3):426-36.

59. Stenman LK, Waget A, Garret C, Briand F, Burcelin R, Sulpice T, et al. Probiotic B420 and prebiotic polydextrose improve efficacy of antidiabetic drugs in mice. Diabetol Metab Syndr. 2015;7(1):75.

60. D'Alessio DA, Denney AM, Hermiller LM, Prigeon RL, Martin JM, Tharp WG, et al. Treatment with the dipeptidyl peptidase-4 inhibitor vildagliptin improves fasting islet-cell function in subjects with type 2 diabetes. J Clin Endocrinol Metab. 2009;94(1):81-8.

61. Mannucci E, Tesi F, Bardini G, Ognibene A, Petracca MG, Ciani S, et al. Effects of metformin on glucagon-like peptide-1 levels in obese patients with and without Type 2 diabetes. Diabetes Nutr Metab. 2004; 17(6):336-42.

\section{Publisher's Note}

Springer Nature remains neutral with regard to jurisdictional claims in published maps and institutional affiliations.
Ready to submit your research? Choose BMC and benefit from:

- fast, convenient online submission

- thorough peer review by experienced researchers in your field

- rapid publication on acceptance

- support for research data, including large and complex data types

- gold Open Access which fosters wider collaboration and increased citations

- maximum visibility for your research: over 100M website views per year

At BMC, research is always in progress.

Learn more biomedcentral.com/submissions 